Results Trends in heavy drinking showed no real increase since the '70s for either men or women. 40-50 years ago, average BMI was within the "normal" range of 20-25, but now it is above this range for most age groups. Childhood overweight and obesity has also dramatically increased since the '70s. Smoking rates have declined with four times less tobacco available in the UK now since 1961. The quality of the UK diet has improved, with reductions in saturated fat and sugar intake, and increases in fruit consumption. Long term trends in physical activity levels were unclear.

Conclusion Falls in cardiovascular disease are due to the improvements in smoking and diet-two of the major risk factors. Trends in heavy drinking have remained fairly constant, but the striking increases in average BMI and childhood obesity threaten to slow or even reverse the declines in cardiovascular mortality, a condition which in 2009 killed a third of the UK population.

\section{SP3-57 DELETERIOUS ORAL HABITS ASSOCIATED TO WEANING IN STUDENTS FROM 3 TO 5 YEARS OLD FROM VITÓRIA/ES: A CASE-CONTROL STUDY}

doi:10.1136/jech.2011.1429760.57

F P de Caxias, M H M de Barros Miotto. ${ }^{*}$ Universidade Federal do Espírito Santo, Espirito Santo, Brazil

Introduction The breastfeeding is extremely important to the general's and stomatognathic system's health of the baby. The aim of this research is to evaluate the existing association between breastfeeding and deleterious oral habits and the prevalence of the habits in children from 03 to 05 years old in Vitória, Espírito Santo. Methods Case-control study, used as parameters to the samples a prevalence of $33 \%$, confidence level of $95 \%$ and margin of error of $5 \%$. The final sample was 903 students, randomised and representative of a total of 9829 children, with complete primary dentition. For the data collection it was used a questionnaire directed to the responsibles. The comparison of the percentages among habits and socioeconomic factors and weaning was tested by Fisher's exact test. To the risk it was used OR. The level of significance was $5 \%$. The statistic package SPSS-Social Package Statistical Science, version 15-was used to this analysis. This research was approved by Comitê de Ética em Pesquisa da UFES (084/2010).

Results $12.4 \%$ present or had presented the habit of finger sucking and $37.7 \%$ the habit of sucking pacifier. There is no statistically significant association of the habit of finger sucking with weaning and children who suffer weaning present approximately 4 times more chance of developing the habit of sucking pacifiers.

Conclusion It is necessary a larger awareness of the mothers about breastfeeding and public politics to create conditions for them to have these healthy choices.

\section{SP3-58 TEETH LOSS IMPACT ON ELDER PEOPLE'S QUALITY OF LIFE}

doi:10.1136/jech.2011.1429760.58

${ }^{1}$ M V de Lima Saintrain, ${ }^{*}{ }^{1}$ E H A de Souza, ${ }^{1}$ A P G F Vieira. ${ }^{1}$ Universidade de Fortaleza, Fortaleza, Ceará, Brazil; ' Universidade de Pernambuco, Recife, Pernambuco, Brazil

Introduction There are scientific evidences which show the health contribution of the quality of life. These measurements have not been well guided towards the free speech of the oral health in the people's life

Objective This study aimed to investigate the impact of the teeth loss on the elder people's quality of life.

Material and Methods Qualitative investigation with descriptive features, which used random out sample as a result of the total edentulous elder people's selection, who live in a institution of
Fortaleza, Brazil. Within 250 residents screened: 72 elderly, completely edentulous, with ages varying from 60 to 79 years old were selected. A semi-structured questionnaire with closed and opened questions in which the last ones were used for the free flow of the interviewee's speech. The content was analysed and codified according to Bardin.

Results $84.7 \%$ had gone to the dentist in order to have exodontics. $81.9 \%$ reported difficulties after losing their teeth. Physical dimensions, characterised by the difficulties to be fed, and social dimensions, due to the interference in the communication with other people were obtained.

Conclusion The tooth loss causes disorder in the individual's quality life, mainly when it affects his well-being and appearance.

\section{SP3-59 ORAL HEALTH OF THE ELDERLY: PREVALENCE OF ORAL LESIONS RELATED TO DELETERIOUS HABITS}

doi:10.1136/jech.2011.1429760.59

${ }^{1} \mathrm{M}$ V de Lima Saintrain,* ${ }^{1} \mathrm{~T}$ G Holanda, ${ }^{1} \mathrm{~T}$ M M Bezerra, ${ }^{1} \mathrm{P}$ C de Almeida. ${ }^{1}$ Universidade de Fortaleza, Fortaleza, Ceará, Brazil; ${ }^{2}$ Universidade Estadual do Ceará, Fortaleza, Ceará, Brazil

Introduction With increasing age, the oral mucosa becomes more susceptible to external stimuli, favouring increased exposure of the individual to aggressive agents. Considering the deleterious habits, the use of alcohol and tobacco represents risk factors for oral lesions. Studies show that the incidence of oral cancer accompanies patterns of tobacco and alcohol consumption.

Objective To investigate the prevalence of oral lesions and relate them to deleterious habits in elderly patients.

Methodology It was a quantitative research conducted at the dental Clinic of UNIFOR, Fortaleza-CE, in the period from 1998 to 2006 The sample consisted of all 756 records of the elderly, and the date were processed by SPSS 15.0.

Results The majority (63.0) were women, age 60-92, mean age $67 \pm 6$. The most significant proportions were: married $(48.4 \%)$ retired $(42.3 \%)$, incomplete primary school $(31.5 \%)$. It was found a prevalence of $18.3 \%$ of oral lesions, with the risk being 1.6 times higher ( $p=0.030$ ) among younger (up to 65 years) and 1.7 times higher among smokers $(p=0.048)$. Although this risk is 1.6 times higher among those who drank alcohol, it was not statistically significant $(\mathrm{p}=0.122)$.

Conclusion The elderly showed a high prevalence of oral lesion, being the youngest, the smokers and the alcohol consumers those most vulnerable to the emergence of these diseases. Dental services need to implement programs of elderly care and health education, essential to clarify the risks and their associations with oral diseases, aiming to promote health.

\section{SP3-60 OLDER PEOPLE'S ORAL HEALTH EVALUATION AND THEIR INTERFERENCE IN DEPRESSION SYMPTOMS}

doi:10.1136/jech.2011.142976o.60

${ }^{1} \mathrm{M}$ V de Lima Saintrai, ${ }^{*} \mathrm{~A}$ V P Guimarães, ${ }^{1} \mathrm{~V}$ A Honório, ${ }^{1} \mathrm{P}$ C de Almeida ${ }^{1}$ Universidade de Fortaleza, Fortaleza, Ceará, Brazil; ${ }^{2}$ Universidade Estadual do ceará, Fortaleza, Ceará, Brazil

Introduction Population's ageing brings problems that confront health systems.

Objective Evaluate the oral health of elderly patients and their interference in depression symptoms.

Methodology Quantitative research applied for elderly of Reference Centers for Social Welfare in Fortaleza. Applied: Geriatric Depression Scale and an identification and epidemiological survey questionnaire. Software SPSS, version 15, computed the data. 
Results 150 patients between 60 and 91 years old (mean 69.37 and $\mathrm{SD} \pm 7169)$ participated. Most patients were women, white race, widowhood, low educational level and family income. 54\% was considered reasonable general health, $14.7 \%$ poor and $51.3 \%$ the same condition as last year. $66 \%$ were edentulous. Highlighted problems: tartar, inflamed gums, cavities, residual root, soft tissue injuries, prosthesis needs. Last year, only $20 \%$ visited the dentist. Main cited diseases: vision, hypertension, hearing, osteoporosis, diabetes mellitus, cardiovascular diseases, rheumatism and cholesterol. $10.7 \%$ were smokers and $69.3 \%$ used drugs. Symptoms of mild and severe depression were detected. Statistical association verified among depression symptoms with: perceived general health $(p=0.003)$, osteoporosis $(p=0.007)$. Depression and oral health: smoking ( $p=0.040)$, dry mouth $(p=0.001)$, burning mouth $(p=0.055)$, pain in the mouth $(p=0.009)$, swelling of the mouth $(p=0.001)$ and using prosthesis $(p=0.005)$.

Conclusion Older people's oral health is poor and most show depressive symptoms. The consequences of teeth condition were significant with depressive problems, providing public health burden that must be considered by public policy.

\section{SP3-61 PRESENT AND PREVIOUS ADOLESCENT PREGNANCY: SOCIOECONOMIC CHARACTERISTICS AND THE EFFECT ON NEWBORN WEIGHT}

doi:10.1136/jech.2011.1429760.61

S Gama, ${ }^{*}$ A P Esteves-Pereira, M do Carmo Leal. ENSP, FIOCRUZ, Rio de Janeiro, Brazil

Introduction The negative effects of pregnancy in adolescence on maternal and child health have been described. However gaps in the literature examination the association between this exposure and outcome remain. The aim of this study was to examine the association between socioeconomic characteristics of three groups of mothers (adolescents ( $<20$ years old), women $\geq 20$ years old with a previous pregnancy during adolescence and women $\geq 20$ years old without a pregnancy during adolescence) and low birth weight $(<2500 \mathrm{~g})$.

Methods 1681 women in the first trimester of pregnancy were interviewed in two cities; follow-up at postpartum was available for 1540. To verify the hypothesis of homogeneity of proportions, $\chi^{2}$ tests were used. To verify the association with low birth weight we conducted crude and adjusted logistic regression analyses with a significance level of 0.05 .

Results Women $\geq 20$ years old with a history of pregnancy in adolescence were more socioeconomically deprived than other groups. Compared to women $\geq 20$ years old without a history of pregnancy in adolescence, women $\geq 20$ years old with a history of pregnancy in adolescence had a lower level of educational attainment [OR 2.68], lower social status [OR 1.96] and lower income [OR 2.71]. Low birth weight was greater in the children of adolescent mothers [OR 1.36]. Conclusions These findings suggest that in Brazil adolescent pregnancy is a social problem and women with a pregnancy in adolescence are socioeconomically disadvantaged through their lives.

\section{SP3-62 AGREEMENT BETWEEN MEDICAL RECORD AND PARENT REPORT FOR EVALUATION OF CHILDHOOD FEBRILE SEIZURES}

doi:10.1136/jech.2011.1429760.62

B Ackerson, ${ }^{*}$ L S Sy, J F Yao, T C Cheetham, T L Jones, S J Jacobsen. Kaiser Permanente Southern California, Pasadena, California, USA

Introduction Little is known about the quality of medical record data used to evaluate febrile seizures despite its influence on vaccine recommendations.
Methods Parent report from phone interviews and mailed questionnaires was compared to abstracted medical records of 110 children with febrile seizures between ages 3 and 60 months Concordance between parent report and medical records for characteristics and predisposing factors of febrile seizures was assessed by per cent total agreement and $\kappa$ statistic. Sensitivity of medical record and parent report was determined setting parent report and medical record as the standard, respectively (Tisnado, 2006).

Results Per cent total agreement between medical record and parent report was good for many variables studied $(62.5 \%$ had $>70 \%$ agreement). However, $\kappa$ was low for all measures $(100 \%$ had $\kappa$ $<0.33)$ For seizure characteristics, parent report was more sensitive than medical record (69.9\% (95\% CI 50.0 to 88.9$)$ and $31.9 \%(95 \%$ CI 18.2 to 45.7), respectively) while both were sensitive for fever (91.0\% (95\% CI 85.4 to 96.6 ) vs $91.9 \%(95 \%$ CI 86.6 to 97.3$)$ respectively). Neither was sensitive for predisposing factors $(62.1 \%$ (95\% CI 41.5 to 81.8$)$ ) and $64.1 \%$ (95\% CI 44.0 to 83.3), respectively). Conclusion These data demonstrate the limitations of using only medical records or parent report to assess febrile seizures. Collection of information from both sources is required to most accurately portray the spectrum of predisposing factors and seizure characteristics of childhood febrile seizures.

\section{SP3-63 FROM PRE-NATAL TO 3 MONTHS OLD: LONGITUDINAL STUDY WITH ADOLESCENT MOTHERS}

doi:10.1136/jech.2011.1429760.63

S Gama, ${ }^{*}$ A P Esteves-Pereira, M do Carmo Leal. ENSP, FIOCRUZ, Rio de Janeiro, Brazil

Introduction The negative effects of pregnancy in adolescence on the mother and child health has been well established, however there are still remaining gaps to more studies to explore this cause-effect relation

Objectives To compare three groups of mothers-adolescents $(<20$ years) and two composed of $\geq 20$-year-old women classified according to past experience of pregnancy during adolescence-with respect to perinatal care.

Methods A sample of 1681 women was interviewed in two cities during the first trimester of pregnancy, a follow-up sample of 1540 at postpartum and 1434 after 3 months. We conducted crude and adjusted logistic regression analysis for each outcome, with a significance level of $5 \%$

Results The most disadvantaged conditions were found among the adolescent's mothers and those with a history of pregnancy in adolescence. In comparison with women who hadn't been pregnant in adolescence, they had, respectively, a higher frequency of inadequate pre-natal-care [OR 1.8 and 1.5], no postpartum visit [OR 1.3 and 1.5], no performance of newborn screening test [OR 2.3 and 2.1] and baby's first doctor visit $>1$-month-old [OR 1.7 and 1.8]. No differences were found regarding breastfeeding at 3-month-old and immunisation. After adjustment for city, educational level and skin colour the association remained statistically significant for pre-natal inadequacy [OR 1.7 and 1.4] and baby's first doctor visit $>1$-monthold [OR 1.5 and 1.6].

Conclusions Previous and current adolescence pregnancy seems to be a risk factor for inadequate healthcare for both women and its offspring.

\section{SP3-64 ASSOCIATION BETWEEN HISTORY OF TUBERCULOSIS AND VEGETARIANISM FROM A NATIONALLY REPRESENTATIVE SURVEY IN INDIA}

doi:10.1136/jech.2011.142976o.64

${ }^{1} \mathrm{P}$ Arora, ${ }^{1} \mathrm{P}$ Jha, ${ }^{1} \mathrm{~N}$ Nagelkerke. ${ }^{1}$ Centre for Global Health Research (CGHR), Li Ka Shing Knowledge Institute, St. Michael's Hospital, Dalla Lana School of Public Health, 